\title{
Polynomial Bases on the Numerical Solution of the Multivariate Discrete Moment Problem
}

\author{
Gergely Mádi-Nagy
}

Dedicated to Professor András Prékopa on his 80th birthday

Received: date / Accepted: date

\begin{abstract}
The multivariate discrete moment problem (MDMP) has been introduced by Prékopa. The objective of the MDMP is to find the minimum and/or maximum of the expected value of a function of a random vector with a discrete finite support where the probability distribution is unknown, but some of the moments are given. The MDMP can be formulated as a linear programming problem, however, the coefficient matrix is very ill-conditioned. Hence, the LP problem usually cannot be solved in a regular way. In the univariate case Prékopa developed a numerically stable dual method for the solution. It is based on the knowledge of all dual feasible bases under some conditions on the objective function. In the multidimensional case the recent results are also about the dual feasible basis structures. Unfortunately, at higher dimensions, the whole structure has not been found under any circumstances. This means that a dual method, similar to Prékopa's, cannot be developed. Only bounds on the objective function value are given, which can be far from the optimum. This paper introduces a different approach to treat the numerical difficulties. The method is based on multivariate polynomial bases. Our algorithm, in most cases, yields the optimum of the MDMP without any assumption on the objective function. The efficiency of the method is tested on several numerical examples.
\end{abstract}

Keywords Discrete moment problem · Multivariate Lagrange interpolation · Linear programming $\cdot$ Expectation bounds Probability bounds

Mathematics Subject Classification (2000) 62H99 $\cdot 90 \mathrm{C} 05 \cdot 65 \mathrm{D} 05$

\section{Introduction}

The multivariate discrete moment problem (MDMP) has been introduced by Prékopa (1992). It is a natural generalization of the so-called univariate discrete moment problem, which

G. Mádi-Nagy

Department of Operations Research, Eötvös University, Pázmány Péter sétány 1/c, Budapest, Hungary, H-1117

E-mail: gergely@cs.elte.hu

Tel.: +36-1-3812169, Fax: +36-1-3812148 
was introduced and studied by Prékopa (1988, 1990a, 1990b) and Samuels and Studden (1989), independently. Samuels and Studden use the classical approach and their method is applicable only to small size problems. Prékopa invented a numerically stable dual simplex algorithm to solve the underlying linear programming problem. This method allows for an efficient solution of large size moment problems as well as for finding closed form sharp bounds. Unfortunately, the dual method of Prékopa could not be generalized to the multivariate case. His method needs the knowledge of all dual feasible bases, but in the multivariate case only a smaller set of them are known. The dual feasible bases provide us with bounds for the MDMP, see Prékopa (1998, 2000), Mádi-Nagy and Prékopa (2004) and Mádi-Nagy (2005, 2009). However, the optimum of the problem usually cannot be found. The aim of this paper is to introduce an algorithm that finds the optimum, usually in a numerically stable way, based on another approach.

The MDMP can be formulated as follows. Let $\boldsymbol{X}=\left(X_{1}, \ldots, X_{s}\right)$ be a random vector and assume that the support of $X_{j}$ is a known finite set $Z_{j}=\left\{z_{j 0}, \ldots, z_{j n_{j}}\right\}$, where $z_{j 0}<\cdots<z_{j n_{j}}, j=1, \ldots, s$. A certain set of the following moments are considered.

Definition 1 The $\left(\alpha_{1}, \ldots, \alpha_{s}\right)$-order power moment of the random vector $\left(X_{1}, \ldots, X_{s}\right)$ is defined as

$$
\mu_{\alpha_{1} \ldots \alpha_{s}}=E\left[X_{1}^{\alpha_{1}} \cdots X_{s}^{\alpha_{s}}\right]
$$

where $\alpha_{1}, \ldots, \alpha_{s}$ are nonnegative integers. The sum $\alpha_{1}+\cdots+\alpha_{s}$ will be called the total order of the moment.

We use the following notation for the (unknown) distribution of $\boldsymbol{X}$ :

$$
p_{i_{1} \ldots i_{s}}=P\left(X_{1}=z_{1 i_{1}}, \ldots, X_{s}=z_{s i_{s}}\right), 0 \leq i_{j} \leq n_{j}, j=1, \ldots, s .
$$

Then the moments can be written in the form

$$
\mu_{\alpha_{1} \ldots \alpha_{s}}=\sum_{i_{1}=0}^{n_{1}} \cdots \sum_{i_{s}=0}^{n_{s}} z_{1 i_{1}}^{\alpha_{1}} \cdots z_{s i_{s}}^{\alpha_{s}} p_{i_{1} \ldots i_{s}} .
$$

Let $Z=Z_{1} \times \cdots \times Z_{s}$ and

$$
f(z), z \in Z
$$

be a function. Let

$$
f_{i_{1} \ldots i_{s}}=f\left(z_{1 i_{1}}, \ldots, z_{s i_{s}}\right) .
$$

The (power) MDMP is to give bounds for

$$
E\left[f\left(X_{1}, \ldots, X_{s}\right)\right]
$$

where the distribution of $\boldsymbol{X}$ (i.e., (1)) is unknown, but known are some of the following moments:

$$
\mu_{\alpha_{1} \ldots \alpha_{s}} \text { for }\left(\alpha_{1} \ldots \alpha_{s}\right) \in H \text {. }
$$

In the literature, several types of sets $H$ can be found. In this paper we consider the case

$$
H=\left\{\left(\alpha_{1}, \ldots, \alpha_{s}\right) \mid 0 \leq \alpha_{j}, \alpha_{j} \text { integer, } \alpha_{1}+\cdots+\alpha_{s} \leq m, j=1, \ldots, s\right\},
$$


where $m$ is a given nonnegative integer. We can formulate the MDMP by the following LP problem:

subject to

$$
\min (\max ) \sum_{i_{1}=0}^{n_{1}} \cdots \sum_{i_{s}=0}^{n_{s}} f_{i_{1} \ldots i_{s}} p_{i_{1} \ldots i_{s}}
$$

$$
\begin{aligned}
& \sum_{i_{1}=0}^{n_{1}} \cdots \sum_{i_{s}=0}^{n_{s}} z_{1 i_{1}}^{\alpha_{1}} \cdots z_{s i_{s}}^{\alpha_{s}} p_{i_{1} \ldots i_{s}}=\mu_{\alpha_{1} \ldots \alpha_{s}} \\
& \text { for }\left(\alpha_{1} \ldots \alpha_{s}\right) \in H \\
& p_{i_{1} \ldots i_{s}} \geq 0, \text { all } i_{1}, \ldots, i_{s} .
\end{aligned}
$$

In problem (4) $p_{i_{1} \ldots i_{s}}, 0 \leq i_{j} \leq n_{j}, j=1, \ldots, s$ are the unknown variables, all other parameters (i.e., the function $f$ and the moments) are given. Let us use the following notation for the compact matrix form of (4) with $H$ of (3):

$$
\begin{gathered}
\text { subject to } \quad \begin{array}{c}
\min (\max ) \quad \boldsymbol{f}^{T} \boldsymbol{p} \\
A \boldsymbol{p}=\boldsymbol{b} \\
\boldsymbol{p} \geq \mathbf{0} .
\end{array}
\end{gathered}
$$

Besides arising in a natural way, the MDMP can be applied in several other fields, e.g., bounding expected utilities (Prékopa and Mádi-Nagy, 2008), solving generalized $s$ dimensional transportation problems (Hou and Prékopa, 2007) and approximating values of multivariate generating functions (Mádi-Nagy and Prékopa, 2007). One of the most popular applications is to bound probabilities of Boolean functions of events. These results are based on the so-called binomial MDMP, see e.g., Mádi-Nagy (2009).

The main problem with the solution of the MDMP (4) is that the coefficient matrix is very ill-conditioned. (It is easy to see that in the univariate case the coefficient matrix is a Vandermonde matrix, which is one of the well-known examples of ill-conditioned matrices.) Hence, in case of the (dual) simplex method in the calculation of the basic solutions and optimality conditions, the numerical inaccuracy is much larger than it was in the input data. This means that if we try to solve the MDMP with regular solvers they will yield not only inaccurate, but wrong results.

This phenomenon can be managed in several ways. One alternative is the use of high precision arithmetic. Its disadvantage is that the running time will be extremely increased. Another, much more elegant way is the mentioned revised dual method of Prékopa (1990b). This method is based on theorems which give the subscript structures of columns of all dual feasible bases. By the aid of the known dual feasible bases, at every iteration in the dual simplex method the following basis can be found combinatorially. Unfortunately, this method works only for the univariate case beside some conditions on the function $f(z)$ in (2). In this paper another approach is introduced. 
Let us consider the following vector:

$$
\boldsymbol{b}(\boldsymbol{z})=\boldsymbol{b}\left(z_{1}, \ldots, z_{s}\right)=\left(\begin{array}{c}
1 \\
z_{1} \\
z_{1}^{2} \\
\vdots \\
z_{1}^{\alpha_{1}} z_{2}^{\alpha_{2}} \ldots z_{s}^{\alpha_{s}} \\
\vdots \\
z_{s}^{m}
\end{array}\right) \text {, where }\left(\alpha_{1}, \ldots, \alpha_{s}\right) \in H .
$$

Regarding the columns of the coefficient matrix $A$ in (5), they can be formulated as

$$
\boldsymbol{a}_{i_{1} \ldots i_{s}}=\boldsymbol{b}\left(z_{1 i_{1}}, \ldots, z_{s i_{s}}\right) .
$$

The right-hand side vector can also be written as

$$
\boldsymbol{b}=E\left(\boldsymbol{b}\left(X_{1}, \ldots, X_{s}\right)\right) .
$$

The idea is the following. The components of $\boldsymbol{b}(\boldsymbol{z})$ of $(6)$ are the monomial basis of the $s$-variate polynomials of degree at most $m$. Let us consider another basis of the $s$-variate polynomials of degree at most $m$ :

$$
p_{0 \ldots 0}(\boldsymbol{z}), p_{1 \ldots 0}(\boldsymbol{z}), \ldots, p_{\alpha_{1} \ldots \alpha_{s}}(\boldsymbol{z}), \ldots, p_{0 \ldots m}(\boldsymbol{z})
$$

Let

$$
\overline{\boldsymbol{b}}(\boldsymbol{z})=\overline{\boldsymbol{b}}\left(z_{1}, \ldots, z_{s}\right)=\left(\begin{array}{c}
p_{0 \ldots 0}(\boldsymbol{z}) \\
p_{1 \ldots 0}(\boldsymbol{z}) \\
\vdots \\
p_{\alpha_{1} \ldots \alpha_{s}}(\boldsymbol{z}) \\
\vdots \\
p_{0 \ldots m}(\boldsymbol{z})
\end{array}\right) \text {, where }\left(\alpha_{1}, \ldots, \alpha_{s}\right) \in H,
$$

and

$$
\overline{\boldsymbol{b}}=E\left(\overline{\boldsymbol{b}}\left(X_{1}, \ldots, X_{s}\right)\right)
$$

The system of linear equations $\bar{A} \boldsymbol{p}=\overline{\boldsymbol{b}}$ is equivalent to the system $A \boldsymbol{p}=\boldsymbol{b}$ of (5), since there exists an invertible matrix $T$ such that

$$
\bar{A}=T A \text { and } \bar{b}=T \boldsymbol{b} .
$$

The aim of the paper is to find out which basis (7) yields a significantly better conditioned matrix $\bar{A}$. By the use of this basis we can solve

$$
\begin{aligned}
& \min (\max ) \boldsymbol{f}^{T} \boldsymbol{p} \\
& \text { subject to } \\
& \bar{A} \boldsymbol{p}=\overline{\boldsymbol{b}} \\
& \boldsymbol{p} \geq \mathbf{0} \text {, }
\end{aligned}
$$

instead of problem (5), in a numerically more stable way. In the following, first the candidates for polynomial bases are introduced with their main properties, and with the 
reasons why they are taken into account. Then a solution algorithm is developed, which is suitable to yield numerically reliable results as well as to indicate the violations of primal and dual infeasibility. Finally, numerical tests are carried out in order to find the basis which yields the best (most reliable) results. Our method is heuristic in the sense that its usefulness is not proven, just analyzed empirically. However, the developed algorithm is very effective in practice, and it is also reliable because it indicates the wrong solution.

The paper is organized as follows. In Section 2 the possible polynomial bases are introduced. In Section 3 the solution algorithm and the testing method are presented. Section 4 is about the numerical experiments. In the first part of this section, conditions of randomly generated basis matrices are investigated. This shows which basis has better numerical properties. In the second part, several MDMPs are solved to illustrate that the bases with better condition numbers really work better in the solutions of practical problems. Section 5 concludes the paper.

\section{Polynomial bases}

In order to present the properties of the following bases, first we need some introduction on condition numbers. The condition number of a matrix can be defined in the following way. Consider the following system of linear equations:

$$
A \boldsymbol{x}=\boldsymbol{b},
$$

where $A$ is a square matrix. Let us imagine that there is an error $\boldsymbol{e}$ in $\boldsymbol{b}$, hence we get a solution with error $\boldsymbol{d}$, i.e.,

$$
A(\boldsymbol{x}+\boldsymbol{d})=\boldsymbol{b}+\boldsymbol{e}
$$

The condition number of the matrix $A$ is the maximum ratio of the relative error in the solution $\boldsymbol{x}$ to the relative error in the right-hand side vector $\boldsymbol{b}$. I. e., the condition number of $A$ is the maximum of the following fraction:

$$
\frac{\|\boldsymbol{d}\| /\|\boldsymbol{x}\|}{\|\boldsymbol{e}\| /\|\boldsymbol{b}\|}=\frac{\left\|A^{-1} \boldsymbol{e}\right\| /\|\boldsymbol{x}\|}{\|\boldsymbol{e}\| /\|\boldsymbol{A} \boldsymbol{x}\|}=\left(\left\|A^{-1} \boldsymbol{e}\right\| /\|\boldsymbol{e}\|\right)(\|A \boldsymbol{x}\| /\|\boldsymbol{x}\|) .
$$

It is easy to see that the maximum of the first and second term is $\left\|A^{-1}\right\|$ and $\|A\|$, respectively. From this follows

Definition 2 The condition number of the quadratic matrix $A$ is

$$
\kappa(A)=\left\|A^{-1}\right\| \cdot\|A\| .
$$

In case of polynomial bases the condition number can be defined in the following way, see e.g., Lyche and Peña (2004). Let $U$ be a finite-dimensional vector space of functions defined on $\Omega \in \mathbb{R}^{s}$ and let $b=\left(b_{1}, \ldots, b_{n}\right)$ be a basis for $U$. Given a function $f=\sum_{i=1}^{n} c_{i} b_{i} \in U$, the condition numbers measure for the sensitivity of $f(\boldsymbol{z})$ to perturbations in the coefficients $\boldsymbol{c}=\left(c_{1}, \ldots, c_{n}\right)$ of $f$. If $g=\sum_{i=1}^{n}\left(1+\delta_{i}\right) c_{i} b_{i}$ is related to $f$ by a relative perturbation $\boldsymbol{\delta}=\left(\delta_{1}, \ldots, \delta_{s}\right)$ in $\boldsymbol{c}$, then for any $\boldsymbol{z} \in \Omega$

$$
|f(\boldsymbol{z})-g(\boldsymbol{z})|=\left|\sum_{i=1}^{n} \delta_{i} c_{i} b_{i}(\boldsymbol{z})\right| \leq\|\delta\|_{\infty} \sum_{i=1}^{n}\left|c_{i} b_{i}(\boldsymbol{z})\right| .
$$


Let

$$
C_{b}(f, \boldsymbol{z})=\sum_{i=1}^{n}\left|c_{i} b_{i}(\boldsymbol{z})\right| .
$$

Definition 3 The polynomial basis $b=\left(b_{1}, \ldots, b_{n}\right)$ for $U$ on $\Omega \in \mathbb{R}^{s}$ has the following type of condition numbers.

$$
\begin{gathered}
\operatorname{cond}(b ; f, \boldsymbol{z})=\frac{C_{b}(f, \boldsymbol{z})}{\|f\|_{\infty}}=\frac{\sum_{i=1}^{n}\left|c_{i} b_{i}(\boldsymbol{z})\right|}{\left\|\sum_{i=1}^{n} c_{i} b_{i}\right\|_{\infty}}, \\
\operatorname{cond}(b ; f)=\sup _{\boldsymbol{z} \in \Omega} \operatorname{cond}(b ; f, \boldsymbol{z}), \\
\operatorname{cond}(b)=\sup _{f \in U} \operatorname{cond}(b ; f) .
\end{gathered}
$$

Above, the so-called Skeel condition numbers are defined, see e.g. Skeel (1979). The $p$-norm condition numbers can be also defined as a ratio of the relative changes in $|f(\boldsymbol{z})-g(\boldsymbol{z})|$ and the relative changes in $p$-norms of the error vector of $\boldsymbol{c}$.

2.1 Bernstein polynomials

Definition 4 The multivariate Bernstein basis polynomials of degree $n$ are defined as

$$
\begin{gathered}
b_{\alpha_{1} \ldots \alpha_{s}}\left(z_{1}, \ldots, z_{s}\right)=\frac{m !}{\alpha_{1} ! \cdots \alpha_{s} !\left(m-\alpha_{1}-\cdots-\alpha_{s}\right) !} z_{1}^{\alpha_{1}} \times \cdots \times z_{s}^{\alpha_{s}} \\
\times\left(1-z_{1}-\cdots-z_{s}\right)^{m-\alpha_{1}-\cdots-\alpha_{s}},
\end{gathered}
$$

where $\left(\alpha_{1}, \ldots, \alpha_{s}\right) \in H$ of $(3)$.

The condition numbers of univariate and multivariate Bernstein polynomial bases are investigated in e.g., Lyche and Scherer (2000, 2002) and Lyche and Peña (2004). The reason why this basis is among the candidates is the following

Theorem 1 (Theorem 5.1 in Lyche and Peña, 2004) Let $\Omega=\left\{\boldsymbol{z} \in \mathbb{R}^{s} \mid z_{1}+\right.$ $\left.\cdots z_{s} \leq 1, z_{i} \geq 0, i=1, \ldots s\right\}$. Let $b$ be the Bernstein basis for the space $U$ of multivariate polynomials of total degree at most $n$. If $u$ is another basis for $U$ of functions which are nonnegative on $\Omega$ and such that

$$
\operatorname{cond}(u ; f, \boldsymbol{z}) \leq \operatorname{cond}(b ; f, \boldsymbol{z})
$$

for each function $f \in U$ evaluated at every value $\boldsymbol{z} \in \Omega$, then $u=b$ up to permutation and positive scaling.

Unfortunately, not all the conditions of the above theorem can be fulfilled. On one hand, in our case bases that are not necessarily non-negative are allowed. On the other hand, the vectors $\boldsymbol{z} \in Z$ spanned a cube instead of the simplex like $\Omega$ in Theorem 1 . Two kinds of rescaling can be considered. One alternative is to put the cube into the simplex. In this case probably a lot of bases exist, which are not better conditioned on the simplex, but better conditioned on the cube. The other alternative is scaling 
the vectors $\boldsymbol{z} \in Z$ to the unit cube $[0,1]^{s}$. We follow this second alternative, hence we consider the scaled Bernstein bases

$$
\begin{gathered}
B_{\alpha_{1} \ldots \alpha_{s}}\left(z_{1}, \ldots, z_{s}\right)=\frac{m !}{\alpha_{1} ! \cdots \alpha_{s} !\left(m-\alpha_{1}-\cdots-\alpha_{s}\right) !}\left(\frac{z_{1}-z_{10}}{z_{1 n_{1}}-z_{10}}\right)^{\alpha_{1}} \times \\
\cdots \times\left(\frac{z_{s}-z_{s 0}}{z_{s n_{s}}-z_{s 0}}\right)^{\alpha_{s}} \times\left(1-\frac{z_{1}-z_{10}}{z_{1 n_{1}}-z_{10}}-\cdots-\frac{z_{s}-z_{s 0}}{z_{s n_{s}}-z_{s 0}}\right)^{m-\alpha_{1}-\cdots-\alpha_{s}},
\end{gathered}
$$

where $\left(\alpha_{1}, \ldots, \alpha_{s}\right) \in H$ of $(3)$.

\subsection{Orthogonal polynomials}

In the following the multivariate generalizations of univariate orthogonal polynomials are considered. First the univariate orthogonal polynomials are introduced.

Definition 5 A set of polynomials $p=\left\{p_{0}, \ldots, p_{n}\right\}$ - where $p_{i}$ has a degree $i, i=$ $0, \ldots, n$ - is called orthogonal on an interval $[a, b]$ (where $a=-\infty$ and $b=+\infty$ are allowed) if for a weight function $w(z)(w(z) \geq 0, z \in[a, b])$, we have $\left\langle p_{i}, p_{j}\right\rangle=0, i \neq j$, where

$$
\langle f, g\rangle=\int_{a}^{b} f(z) g(z) w(z) d z .
$$

Let

$$
c_{i}=\left\langle p_{i}, p_{i}\right\rangle, i=0,1,2, \ldots
$$

If $c_{i}=1$ for all $i$, then $p$ is a set of orthonormal polynomials.

Some well-known properties of orthogonal polynomials are stated next. The set $p$ is a basis of the space of the polynomials of degree at most $m$. Each polynomial in an orthogonal set $p$ has minimal norm among all polynomials with the same degree and leading coefficient. All roots of a polynomial in an orthogonal set $p$ are real, distinct, and strictly inside the interval of orthogonality. All orthogonal polynomials satisfy a three-term recurrence:

$$
p_{0}(z)=a_{0}, p_{1}(z)=d_{0} z+b_{0}, p_{i+1}(z)=d_{i}\left(z-b_{i}\right) p_{i}(z)-a_{i} p_{i-1}(z),
$$

where $a_{i}, d_{i} \neq 0$ for all $i$.

The following three, so-called Jacobi-like, sets of orthogonal polynomials are considered: Legendre polynomials, first- and second-kind Chebyshev polynomials. The main reasons for the choice are that they have special roles in univariate interpolation, detailed below, and on the other hand all of them have the same, finite interval of orthogonality: $[-1,1]$. The properties, and implicitly the definitions, are listed in the following table:

\begin{tabular}{lccccc}
\hline Type & $w(z)$ & $c_{j}$ & $d_{j}$ & $b_{j}$ & $a_{j}$ \\
\hline Legendre & 1 & $\frac{2}{2 j+1}$ & $\frac{2 j+1}{j+1}$ & 0 & $\frac{j}{j+1}$ \\
First-kind Chebyshev & $\frac{1}{\sqrt{1-z^{2}}}$ & $\pi, c_{0}=\frac{\pi}{2}$ & $2, d_{0}=1$ & 0 & 1 \\
Second-kind Chebyshev & $\sqrt{1-z^{2}}$ & $\pi / 2$ & 2 & 0 & 1
\end{tabular}


The classical results on the condition numbers of Vandermonde-like matrices - i.e., matrices of type $\bar{A}$ of (9) in the univariate case - can be found in Gautschi (1983). The main idea can be illustrated by the simplest case, where the nodes $\left\{z_{11}, \ldots, z_{1 n_{1}}\right\}=$ $Z_{1}=Z$ are the roots of the polynomial $p_{n_{1}} \in p$. Consider the following

Theorem 2 (Discrete orthogonality property) Let $\left\{z_{11}, \ldots, z_{1 n_{1}}\right\}$ be the roots of $p_{n_{1}} \in p$ of Definition 5. Then there exist the so called Christoffel numbers $\lambda_{1}, \ldots, \lambda_{n_{1}}$ such that

$$
\sum_{i=1}^{n_{1}} \lambda_{i} p_{m}\left(z_{1 i}\right) p_{k}\left(z_{1 i}\right)=\delta_{m k}, \text { for all } k, m<n_{1}
$$

Proof See e.g., Gautschi (1968).

Thus, if we consider the Frobenius norm, i.e., $\|A\|_{F}=\sqrt{\operatorname{tr}\left(A^{T} A\right)}$, in the condition number of $\bar{A}$ we get the following formula:

$$
\kappa_{F}(\bar{A})=\left\|\bar{A}^{-1}\right\|_{F} \cdot\|\bar{A}\|_{F}=\sqrt{\sum_{i=1}^{n_{1}} \frac{1}{\lambda_{i}}} \cdot \sqrt{\sum_{i=1}^{n_{1}} \lambda_{i}} .
$$

The zero places of Legendre polynomials and first- and second-kind Chebyshev polynomials have a special role in univariate Lagrange interpolation. By the use of them, the Lebesgue constant can be kept in a moderate value even in case of several nodes, see Blyth, Luo and Pozrikidis (2006). In the univariate case, nearly optimally conditioned Vandermonde matrices can be found if the nodes are chosen as the zero places of the first-kind Chebyshev polynomials, see Li (2006).

There are several other theorems on the condition numbers of Vandermonde-like matrices in connection with orthogonal polynomials, however, these results usually focus on the optimal positions of the interpolation nodes yielding better conditioned Vandermonde-type matrices. Unfortunately, in case of the MDMP, we do not know the positions of the points corresponding to the columns of the basis matrix, and additionally they change at every iteration. Hence, it has to be analyzed, at least empirically, whether their good properties keep the basis matrices numerically treatable.

The multivariate counterparts of the univariate orthogonal polynomials can be constructed in the following way. The products of the univariate polynomials are considered, i.e. the set of the corresponding $s$-variate polynomials are

$$
p_{\alpha_{1} \ldots \alpha_{s}}\left(z_{1}, \ldots, z_{s}\right)=p_{\alpha_{1}}\left(z_{1}\right) \times \cdots \times p_{\alpha_{s}}\left(z_{s}\right)
$$

where $\left(\alpha_{1}, \ldots, \alpha_{s}\right) \in H$ of $(3)$. It is easy to see that the above polynomials are also orthogonal regarding the integral on the cube $I^{s}$ with the weight function $w\left(z_{1}\right) \times \cdots \times$ $w\left(z_{s}\right)$, where $I$ is the orthogonality interval of the univariate polynomials. This means that the set (10) is also a basis of the space of the $s$-variate polynomials of degree at most $m$.

In the following the multivariate counterparts of the Legendre, first- and secondkind Chebyshev polynomials are considered. The orthogonality interval at each polynomial is $[-1,1]$. Hence, the values of each component of $Z$ have to be scaled to the interval $[-1,1]$. This leads to the following formulae. 
2.2.1 Legendre polynomials

The following multivariate Legendre polynomials are considered:

$$
P_{\alpha_{1} \ldots \alpha_{s}}\left(z_{1}, \ldots, z_{s}\right)=P_{\alpha_{1}}\left(\frac{2 z_{1}-\left(z_{10}+z_{1 n_{1}}\right)}{z_{1 n_{1}}-z_{10}}\right) \times \cdots \times P_{\alpha_{s}}\left(\frac{2 z_{s}-\left(z_{s 0}+z_{s n_{s}}\right)}{z_{s n_{s}}-z_{s 0}}\right)
$$

where $\left(\alpha_{1}, \ldots, \alpha_{s}\right) \in H$ of $(3)$ and $P_{\alpha}(z)$ is the $\alpha$ th univariate Legendre polynomial.

\subsubsection{First-kind Chebyshev polynomials}

The multivariate first-kind Chebyshev polynomials are defined as

$$
T_{\alpha_{1} \ldots \alpha_{s}}\left(z_{1}, \ldots, z_{s}\right)=T_{\alpha_{1}}\left(\frac{2 z_{1}-\left(z_{10}+z_{1 n_{1}}\right)}{z_{1 n_{1}}-z_{10}}\right) \times \cdots \times T_{\alpha_{s}}\left(\frac{2 z_{s}-\left(z_{s 0}+z_{s n_{s}}\right)}{z_{s n_{s}}-z_{s 0}}\right),
$$

where $T_{\alpha}(z)$ is the $\alpha$ th univariate first-kind Chebyshev polynomial.

\subsubsection{Second-kind Chebyshev polynomials}

The multivariate second-kind Chebyshev polynomials are

$U_{\alpha_{1} \ldots \alpha_{s}}\left(z_{1}, \ldots, z_{s}\right)=U_{\alpha_{1}}\left(\frac{2 z_{1}-\left(z_{10}+z_{1 n_{1}}\right)}{z_{1 n_{1}}-z_{10}}\right) \times \cdots \times U_{\alpha_{s}}\left(\frac{2 z_{s}-\left(z_{s 0}+z_{s n_{s}}\right)}{z_{s n_{s}}-z_{s 0}}\right)$,

where $U_{\alpha}(z)$ is the $\alpha$ th univariate second-kind Chebyshev polynomial.

\section{Solution algorithm and testing method}

The conversion between problem (5) and (9) is also an ill-conditioned problem in most cases, see e.g., Farouki (2000). Hence, the following algorithm will be considered for the solution of the MDMP (4).

\section{Solution Algorithm}

Step 1. Execution of the basis transformation from problem (5) to problem (9) by the use of high precision arithmetic.

Step 2. Solution of problem (9) by a regular LP solver using dual simplex method.

Step 3. Getting the subscripts of the columns of the optimal basis. Checking the primal and dual feasibility by the use of high precision arithmetic with problem (5). Calculating the objective function value.

High precision arithmetic is applied in the above algorithm, too. However, we use it only at the first and last step, i.e., in two iterations. This produces much less running time comparing to the (dual) simplex method where high precision arithmetic should be used at each iteration.

Our aim is to find polynomial bases where the condition numbers of the basis matrices of $\bar{A}$ in (9) are relatively small. This leads to get, on one hand, more reliable, 
on the other hand, better bounds on the objective function. The $\infty$-norm condition numbers are considered, those indicate the rate of $\infty$-norm relative errors in the solution vector to the $\infty$-norm relative errors in the moment vector. This measures not only the reliability of the optimal solution, but also implies the quality of the iterations. I. e., in case of high condition numbers, the small positive component can be calculated as a negative one and vice versa, and this leads to a wrong choice of incoming and outcoming basis variables.

Unfortunately, we do not know in advance which columns of $\bar{A}$ form a basis used in the (dual) simplex method. Hence, in Example 1 the average condition numbers of randomly chosen bases matrices are calculated. We expect that the polynomial basis with the smallest average condition number yields the most reliable result.

This idea is tested in practice by the following method. The same problem is solved with several bases, introduced in the previous section. The results are compared regarding the

1. primal and dual feasibility of the "optimal" bases, yielded by the solver (checking by the use of high precision arithmetic),

2. the $\infty$-norm condition number of the "optimal" basis matrix in problem (9),

3. the "optimal" objective function values.

The phrase "optimal" (within quotation marks) means that although the solver yields the basis as an optimal one, in some cases, it is only dual feasible. Hence, it gives only a lower (upper) bound on the objective function value in case of min ( $\max$ ) problems. The comparison of the "optimal" objective function values shows which polynomial basis yields the best bounds on the objective function value.

Part 2 of the method enables us to compare the condition number of the "optimal" basis matrix with the average condition numbers of Example 1. It would be more accurate to check the condition numbers of the bases of all iterations, however, it cannot be executed within acceptable running time.

In the numerical test of Section 4 Wolfram's Mathematica (2010) is used for the high precision arithmetic calculations of Step 1 and 3 in the solution algorithm. The solver in Step 2 is the ILOG CPLEX 9 (2010).

\section{Numerical experiments}

First, the basis matrices of (9) are simulated by random choices of the nodes corresponding to the column vectors. The condition numbers are tested for each basis candidate.

Example 1 Let $\left(\begin{array}{c}m+s \\ s\end{array}\right)$ points of the $[0,1]^{s}$ cube be generated randomly by the use of uniform distribution. We construct a quadratic matrix with the columns $\overline{\boldsymbol{b}}$ of (8) at the generated points. If the matrix is non-singular then we put it into the sample. We work on 100 element samples and we calculate the infinity-norm condition numbers of the matrices, for each polynomial basis, and take their average. In case of $s=2$ we get the following results: 


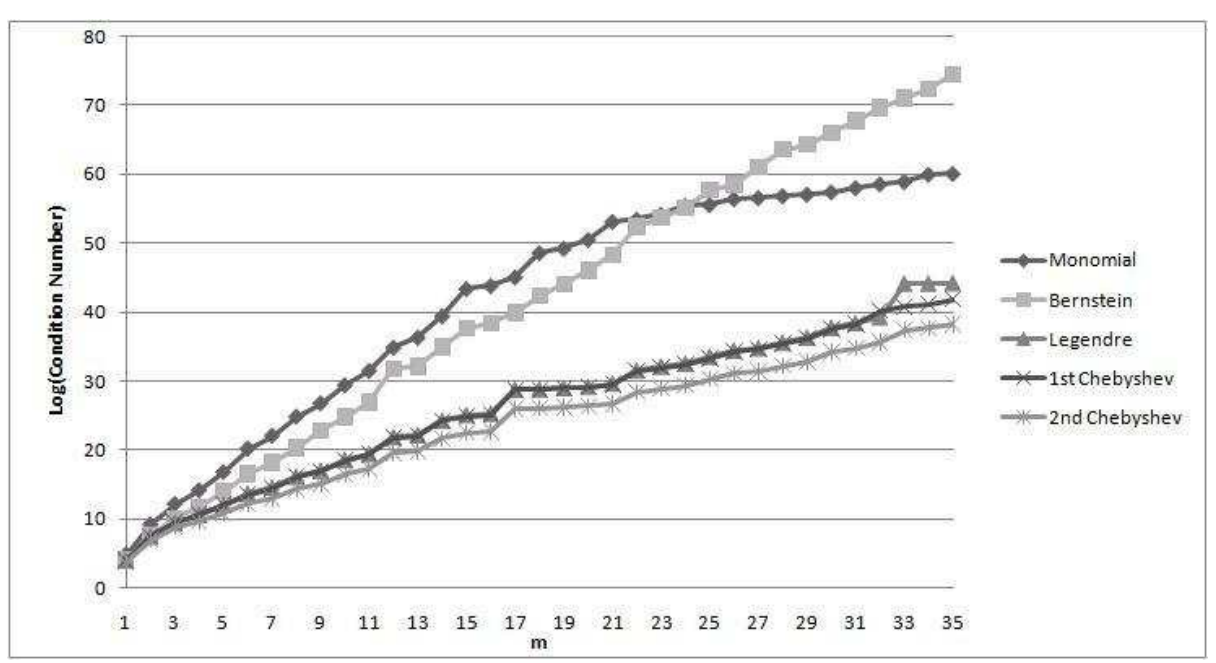

In case of $s=3$ the results are similar:

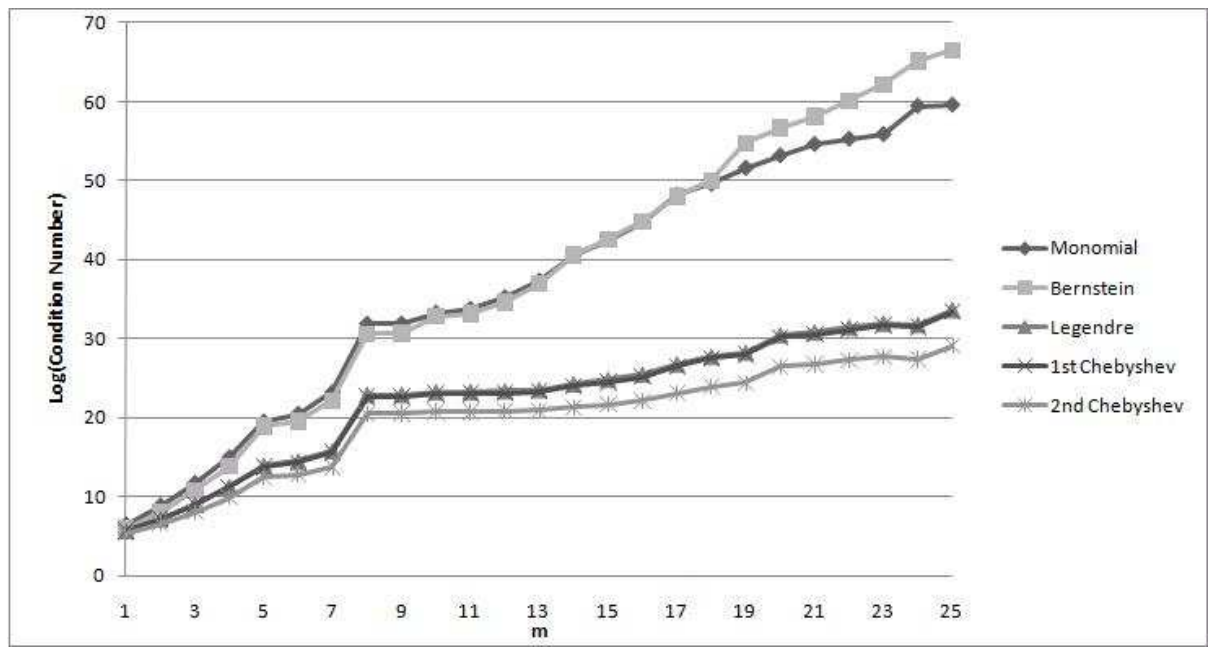

It can be seen, that although all condition numbers increase exponentially, the orthogonal polynomials yield about the square-root of the condition numbers corresponding to the monomial and Bernstein polynomials. This shows that the use of orthogonal polynomials causes dramatically better numerical performance. Note, that in both cases the second-kind Chebyshev polynomial yields the lowest condition numbers.

Example 1 suggests the use of orthogonal bases. In the following we illustrate on the solutions of the MDMPs what kind of numerical troubles can arise. Those examples also illustrate the efficiency of orthogonal bases.

Four problems - with several values of the maximum order $m$ - are solved by the algorithm of Section 3 based on the polynomial bases introduced in Section 2. 
Example 2 Let $Z=\{0,1, \ldots, 15\}^{3}$ and the moments be generated by the uniform distribution on $Z$. Let

$$
f\left(z_{1}, z_{2}, z_{3}\right)=\left\{\begin{array}{l}
0 \text { if }\left(z_{1}, z_{2}, z_{3}\right)=(0,0,0) \\
1 \text { otherwise }
\end{array}\right.
$$

This means that the corresponding MDMP yields bounds for the probability

$$
E\left[f\left(z_{1}, z_{2}, z_{3}\right)\right]=P\left(X_{1}+X_{2}+X_{3}>0\right) .
$$

The results are the following. For the minimum problem:

\begin{tabular}{c|ccccc}
$m$ & Monomial & Bernstein & Legendre & 1st Chebyshev 2 nd Chebyshev \\
\hline \hline 1 & 0.27419355 & 0.27419355 & 0.27419355 & 0.27419355 & 0.27419355 \\
2 & 0.53125000 & 0.53125000 & 0.53125000 & 0.53125000 & 0.53125000 \\
3 & 0.53981855 & 0.53981855 & 0.53981855 & 0.53981855 & 0.53981855 \\
4 & 0.58464908 & 0.58464908 & 0.58464908 & 0.58464908 & 0.58464908 \\
5 & 0.61448581 & 0.61448581 & 0.61448581 & 0.61448581 & 0.61448581 \\
6 & 0.64548271 & 0.64548271 & 0.64548271 & 0.64548271 & 0.64548271
\end{tabular}

For the maximum problem:

\begin{tabular}{c|ccccc}
$m$ & Monomial & Bernstein & Legendre & 1st Chebyshev 2nd Chebyshev \\
\hline \hline 1 & 1.00000000 & 1.00000000 & 1.00000000 & 1.00000000 & 1.00000000 \\
2 & 1.00000000 & 1.00000000 & 1.00000000 & 1.00000000 & 1.00000000 \\
3 & 0.99543011 & 0.99543011 & 0.99543011 & 0.99543011 & 0.99543011 \\
4 & $\mathbf{0 . 9 8 5 7 4 2 1 4}$ & $\mathbf{0 . 9 8 5 7 4 2 1 4}$ & 0.98574214 & 0.98574214 & 0.98574214 \\
5 & 0.98453738 & 0.98453738 & 0.98453738 & 0.98453738 & 0.98453738 \\
6 & 0.95817859 & 0.95817859 & 0.95817859 & 0.95817859 & 0.95817859
\end{tabular}

The results typeset in boldface are dual infeasible solutions, the magnitude of violations are $-5.82 e-7$ and $-8.22 e-7$, respectively. The infinity-norm condition numbers of the optimal basis matrices of the $\mathrm{min} / \max$ problems are:

\begin{tabular}{c||c|c|c|c|c}
$m$ & Monomial & Bernstein & Legendre & 1st Chebyshev & 2nd Chebyshev \\
\hline \hline 1 & $1 E+01 / 1 E+01$ & $7 E+00 / 7 E+00$ & $4 E+00 / 4.00 E+00$ & $4 E+00 / 4 E+00$ & $4 E+00 / 4 E+00$ \\
2 & $4 E+04 / 4 E+03$ & $9 E+02 / 4 E+02$ & $2 E+02 / 1 E+01$ & $2 E+02 / 1 E+01$ & $2 E+02 / 9 E+01$ \\
3 & $1 E+06 / 3 E+05$ & $3 E+03 / 3 E+03$ & $1 E+03 / 4 E+02$ & $2 E+03 / 5 E+02$ & $1 E+03 / 4 E+02$ \\
4 & $1 E+08 / 6 E+06$ & $2 E+05 / 4 E+05$ & $3 E+02 / 8 E+02$ & $4 E+02 / 4 E+02$ & $7 E+02 / 6 E+03$ \\
5 & $2 E+10 / 2 E+11$ & $1 E+06 / 6 E+06$ & $1 E+05 / 1 E+06$ & $8 E+04 / 1 E+06$ & $4 E+04 / 1 E+06$ \\
6 & $8 E+10 / 1 E+11$ & $1 E+07 / 2 E+07$ & $1 E+05 / 2 E+04$ & $2 E+04 / 1 E+04$ & $3 E+04 / 1 E+04$
\end{tabular}

In this case, essentially, all the problems could be solved. However, the condition number can be reduced dramatically by the use of orthogonal polynomials.

Example 3 Let $Z=\{0,1, \ldots, 10\} \times\{0,1, \ldots, 20\} \times\{0,1, \ldots, 30\}$ and the moments be generated in the following way. Let $X, Y_{1}, Y_{2}$ and $Y_{3}$ be random variables with Poisson distribution, with the parameters $\lambda=0.1,0.2,0.3,0.05$, respectively. The moments are generated by the random vector

$$
\left(\min \left(X+Y_{1}, 10\right), \min \left(X+Y_{2}, 20\right), \min \left(X+Y_{3}, 30\right)\right) .
$$

Let

$$
f\left(z_{1}, z_{2}, z_{3}\right)=\sin \left(z_{1}+z_{2}+z_{3}\right) .
$$


The results of the minimum problem are the following:

\begin{tabular}{c|ccccc}
$m$ & Monomial & Bernstein & Legendre & 1st Chebyshev 2 nd Chebyshev \\
\hline \hline 1 & -0.16301713 & -0.16301713 & -0.16301713 & -0.16301713 & -0.16301713 \\
2 & 0.20039622 & 0.20039622 & 0.20039622 & 0.20039622 & 0.20039622 \\
3 & 0.25350547 & 0.25350547 & 0.25350547 & 0.25350547 & 0.25350547 \\
4 & $\underline{0.27167527}$ & $\underline{0.26575235}$ & $\underline{0.27316847}$ & 0.27315366 & 0.27206079 \\
5 & $\underline{0.26469815}$ & $\underline{0.28452612}$ & $\underline{0.28228435}$ & 0.28214106 & 0.28526692 \\
6 & $\underline{0.28393933}$ & $\underline{0.28696505}$ & $\underline{0.28816140}$ & 0.28789129 & 0.28822397
\end{tabular}

The results of the maximum problem:

\begin{tabular}{c|ccccc}
$m$ & Monomial & Bernstein & Legendre & 1st Chebyshev 2nd Chebyshev \\
\hline \hline 1 & 0.71525034 & 0.71525034 & 0.71525034 & 0.71525034 & 0.71525034 \\
2 & 0.47997864 & 0.47997864 & 0.47997864 & 0.47997864 & 0.47997864 \\
3 & 0.31651723 & 0.31651723 & 0.31651723 & 0.31651723 & 0.31651723 \\
4 & 0.31049303 & 0.31108264 & 0.31197905 & 0.31242441 & 0.31244058 \\
5 & $\underline{0.30509224}$ & 0.30263923 & 0.30359098 & 0.30272386 & 0.30352714 \\
6 & 0.29666626 & 0.29609722 & 0.29760208 & 0.29873506 & 0.29855087
\end{tabular}

- The underlined results are not solutions of the system of linear equations $\bar{A} \boldsymbol{p}=\overline{\boldsymbol{b}}$ in (9), hence these results have no meaning. (The solver considers the system of inequalities with zero upper bounds on the slack variables.) The infeasible slack variables have the order of magnitude $10^{-7}$ in case of the Legendre polynomial, however, in case of monomial basis they can be about 60 , which yields very contradictory results.

- The results typeset in italics are not primal feasible, hence they give lower/upper bounds on the objective function of the $\min / \max$ problem.

- It can be seen that the minimum problem can be solved (bounded) only by the aid of the Chebyshev polynomials.

The condition numbers of the optimal bases:

\begin{tabular}{|c|c|c|c|c|c|}
\hline$m$ & Monomial & Bernstein & Legendre & 1st Chebyshev & 2nd Chebyshev \\
\hline 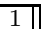 & -01 & +02 & $2 . E+02$ & $4 . E+01 / 2 . E+02$ & $\overline{3 . E+02}$ \\
\hline 2 & & & & & \\
\hline 3 & $3 . E+0$ & & 2. $E+$ & 2. $E+C$ & 1.E \\
\hline 4 & 9. $E+C$ & 3. $E+$ & 1. $E+$ & $4 . E+$ & $4 . E+$ \\
\hline 5 & $2 . E+1$ & 3. $E+09 / 2$ & 7. $E+06 / 7$. & $3 . E+06 / 7 . E+06$ & $2 . E+07 / 2 . E+07$ \\
\hline 6 & $|1 \cdot E+16 / 2 \cdot E+12|$ & $|1 . E+10 / 4 . E+10|$ & $|3 . E+07 / 2 . E+08|$ & $|2 . E+07 / 2 . E+07|$ & $6 . E+07 / 4 . E+07$ \\
\hline
\end{tabular}

The condition numbers are also much lower in case of the orthogonal polynomials, in case of higher values of $m$.

Example 4 Let $Z=\{0,1, \ldots, 100\}^{2}$ and the moments be generated in the following way. Let $X, Y_{1}, Y_{2}$ be random variables with Poisson distribution, with the parameters $\lambda=1,2,3$, respectively. The moments are generated by the random vector

$$
\left(\min \left(X+Y_{1}, 100\right), \min \left(X+Y_{2}, 100\right)\right) .
$$

Let

$$
f\left(z_{1}, z_{2}\right)=\left\{\begin{array}{l}
0 \text { if } z_{1}+z_{2}<6 \\
1 \text { otherwise }
\end{array}\right.
$$


This means that the corresponding MDMP yields bounds for the probability

$$
E\left[f\left(z_{1}, z_{2}\right)\right]=P\left(X_{1}+X_{2} \geq 6\right) .
$$

The results of the minimum problem are the following:

\begin{tabular}{c|ccccc}
$m$ & Monomial & Bernstein & Legendre & 1st Chebyshev 2 nd Chebyshev \\
\hline \hline 1 & 0.01025641 & 0.01025641 & 0.01025641 & 0.01025641 & 0.01025641 \\
2 & 0.30952381 & 0.30952381 & 0.30952381 & 0.30952381 & 0.30952381 \\
3 & 0.34199134 & 0.34199134 & 0.34199134 & 0.34199134 & 0.34199134 \\
4 & $\underline{0.34344014}$ & $\underline{0.34402941}$ & 0.34393909 & 0.34393909 & 0.34394296 \\
5 & $\underline{0.34206839}$ & $\underline{0.35869158}$ & $\underline{0.38154787}$ & 0.38929061 & 0.38739693 \\
6 & $\underline{0.38881997}$ & $\underline{0.38274396}$ & 0.39145364 & 0.39087842 & 0.39136995 \\
7 & $\underline{0.36947755}$ & $\underline{0.38539228}$ & 0.39028745 & 0.39047003 & 0.39058113 \\
8 & $\underline{0.38838059}$ & $\underline{0.41361558}$ & 0.39650524 & 0.38974752 & 0.38956735
\end{tabular}

The results of the maximum problem:

\begin{tabular}{c|ccccc}
$m$ & Monomial & Bernstein & Legendre & 1st Chebyshev 2 nd Chebyshev \\
\hline \hline 1 & 1.00000000 & 1.00000000 & 1.00000000 & 1.00000000 & 1.00000000 \\
2 & 1.00000000 & 1.00000000 & 1.00000000 & 1.00000000 & 1.00000000 \\
3 & 0.94978632 & 0.94978632 & 0.94978632 & 0.94978632 & 0.94978632 \\
4 & 0.94994380 & $\underline{0.94994464}$ & 0.94994380 & 0.94994380 & 0.94994380 \\
5 & 0.93685650 & $\underline{0.93639774}$ & 0.93674363 & 0.93677600 & 0.93695911 \\
6 & 0.89265249 & $\underline{0.92212156}$ & 0.90048010 & 0.92006619 & 0.88778247 \\
7 & $\underline{0.858758376}$ & $\underline{0.88899961}$ & 0.89090362 & 0.88902196 & 0.88883977 \\
8 & 0.86793966 & 0.85465033 & 0.85633780 & 0.87516401 & 0.85490465
\end{tabular}

For higher values of $m$, both the minimum and maximum problems can be solved only by the aid of orthogonal polynomials. The condition numbers of the optimal bases:

\begin{tabular}{|c|c|c|c|c|c|}
\hline$m \|$ & Monomial & Bernstein & Legendre & 1st Chebyshev & 2nd Chebyshev \\
\hline 1 & $7 . E+01 / 4 . E+01$ & $+01 / 3 . E+02$ & $+01 / 2 . E+$ & $2 . E+01 / 2 . E+02$ & $2 . E+01 / 2 . E+02$ \\
\hline 2 & 4. $E+$ & 04 & $04 / 0 . E+$ & $4 . E+04 / 6 . E+$ & $04 / 9 . E+03$ \\
\hline 3 & $2 . E+04 / 2 . E+04$ & $4 . E+06 / 7 . E+06$ & 9. $E+05 / 1 . E+06$ & $9 . E+05 / 9 . E+05$ & 2. $E+06 / 2 . E+06$ \\
\hline 4 & $2 . E+11 / 6 . E+09$ & $2 . E+06 / 1 . E+08$ & $2 . E+06 / 2 . E+06$ & $2 . E+06 / 5 . E+06$ & $2 . E+07 / 1 . E+08$ \\
\hline 5 & $2 . E+14 / 4 . E+11$ & $2 \cdot E+10 / 3 . E+10$ & 7. $E+08 / 7 . E+08$ & $4 . E+09 / 4 . E+08$ & $3 \cdot E+11 / 2 \cdot E+09$ \\
\hline 6 & $2 . E+17 / 3 . E+14$ & 1. $E+10 / 8 . E+10$ & 8. $E+09 / 2 . E+10$ & 7. $E+08 / 4 . E+09$ & $1 . E+10 / 6 . E+10$ \\
\hline 7 & $6 . E+20 / 2 . E$ & $+10 / 2 . E+12$ & $1 . E+09 / 2 . E+09$ & $2 . E+08 / 3 . E+10$ & $1 . E+09 / 2 . E+10$ \\
\hline 8 & $6 . E+$ & $. E+$ & $-09 / 8 . E+$ & $. E+09$ & -09 \\
\hline
\end{tabular}

It can be seen that the best results as well as the lowest condition numbers correspond to the Chebyshev polynomials.

Example 5 Let $Z=\{0,1, \ldots, 100\}^{2}$ again and the moments be generated by the use of uniform distribution on $Z$. Let

$$
f\left(z_{1}, z_{2}\right)=\operatorname{Exp}\left(z_{1} / 50+z_{2} / 200+z_{1} z_{2} / 10000\right)
$$


The results of the minimum problem are the following:

\begin{tabular}{c|ccccc}
$m$ & Monomial & Bernstein & Legendre & 1st Chebyshev 2 nd Chebyshev \\
\hline \hline 1 & 3.97437726 & 3.97437726 & 3.97437726 & 3.97437726 & 3.97437726 \\
2 & 5.27052361 & 5.27052361 & 5.27052361 & 5.27052361 & 5.27052361 \\
3 & 5.91689642 & 5.91689642 & 5.91689642 & 5.91689642 & 5.91689642 \\
4 & $\mathbf{6 . 0 7 9 8 0 3 9 1}$ & $\mathbf{6 . 0 7 9 8 0 3 9 1}$ & $\mathbf{6 . 0 7 9 8 0 3 9 1}$ & $\mathbf{6 . 0 7 9 8 0 3 9 1}$ & $\mathbf{6 . 0 7 9 8 0 3 9 1}$ \\
5 & $\mathbf{6 . 1 3 0 1 4 5 0 9}$ & $\mathbf{6 . 1 3 0 1 4 4 9 7}$ & $\mathbf{6 . 1 3 0 1 4 4 9 7}$ & $\mathbf{6 . 1 3 0 1 4 4 9 8}$ & $\mathbf{6 . 1 3 0 1 4 4 9 7}$ \\
6 & $\mathbf{6 . 1 4 0 3 9 4 8 9}$ & $\mathbf{6 . 1 4 0 3 9 4 9 1}$ & $\mathbf{6 . 1 4 0 3 9 4 8 9}$ & $\mathbf{6 . 1 4 0 3 9 4 8 9}$ & $\mathbf{6 . 1 4 0 3 9 4 8 9}$ \\
7 & $\mathbf{6 . 1 4 2 6 8 7 2 5}$ & $\mathbf{6 . 1 4 2 6 8 7 4 1}$ & $\mathbf{6 . 1 4 2 6 8 7 2 5}$ & $\mathbf{6 . 1 4 2 6 8 7 2 4}$ & $\mathbf{6 . 1 4 2 6 8 7 2 5}$ \\
8 & $\mathbf{6 . 1 4 3 0 8 4 2 6}$ & $\mathbf{6 . 1 4 3 0 8 4 1 9}$ & $\mathbf{6 . 1 4 3 0 8 4 2 1}$ & $\mathbf{6 . 1 4 3 0 8 4 2 2}$ & $\mathbf{6 . 1 4 3 0 8 4 2 3}$
\end{tabular}

The results of the maximum problem:

\begin{tabular}{c|ccccc}
$m$ & Monomial & Bernstein & Legendre & 1st Chebyshev 2 nd Chebyshev \\
\hline \hline 1 & 17.05772598 & 17.05772598 & 17.05772598 & 17.05772598 & 17.05772598 \\
2 & 7.72492540 & 7.72492540 & 7.72492540 & 7.72492540 & 7.72492540 \\
3 & 6.63947797 & 6.63947797 & 6.63947797 & 6.63947797 & 6.63947797 \\
4 & 6.22373114 & 6.22373114 & 6.22373114 & 6.22373114 & 6.22373114 \\
5 & $\mathbf{6 . 1 6 2 7 6 6 8 8}$ & 6.16276688 & 6.16276688 & 6.16276688 & $\mathbf{6 . 1 6 2 7 6 6 8 8}$ \\
6 & $\mathbf{6 . 1 4 6 2 6 0 9 6}$ & $\mathbf{6 . 1 4 6 2 6 0 8 5}$ & $\mathbf{6 . 1 4 6 2 6 0 8 8}$ & $\mathbf{6 . 1 4 6 2 6 0 9 6}$ & $\mathbf{6 . 1 4 6 2 6 0 9 6}$ \\
7 & $\mathbf{6 . 1 4 3 7 6 9 4 9}$ & $\mathbf{6 . 1 4 3 7 6 9 5 0}$ & $\mathbf{6 . 1 4 3 7 6 9 4 9}$ & $\mathbf{6 . 1 4 3 7 6 9 5 0}$ & $\mathbf{6 . 1 4 3 7 6 9 4 8}$ \\
8 & $\mathbf{6 . 1 4 3 2 5 8 0 8}$ & $\mathbf{6 . 1 4 3 2 5 8 0 9}$ & $\mathbf{6 . 1 4 3 2 5 8 0 2}$ & $\mathbf{6 . 1 4 3 2 5 7 8 7}$ & $\mathbf{6 . 1 4 3 2 5 7 8 5}$
\end{tabular}

Unfortunately, this problem cannot even be solved by the aid of orthogonal polynomials, for higher values of $m$. The order of magnitude of the violations in the dual feasibility is about $10^{-7}$ in each case. Note, that in case of our solver, CPLEX 9, the optimality tolerance can be changed to $10 e-09$ from the default value $10 e-06$, and in this case the optimal solution can be found. However, this example illustrates the usefulness of the checking part of our solution algorithm: it detects the infeasibilities even if they are under the numerical tolerances of the solver. It is important if the condition number of the basis matrix is high, like in case of the MDMP.

The condition numbers of the optimal bases:

\begin{tabular}{|c|c|c|c|c|c|}
\hline$m$ & Monomial & Bernstein & Legendre & 1st Chebyshev & 2nd Chebyshev \\
\hline & $\overline{2 . E+04 / 2 . E+02}$ & & $\overline{+02 / 3 . E+0}$ & 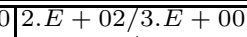 & $2 . E+02 / 5 . E+00$ \\
\hline & 3. $E+C$ & & & & \\
\hline & $6 . E+0$ & 9. $E+$ & $E+02$ & $2 . E+02 / 2 . E+02$ & $3 . E+03 / 3 . E+03$ \\
\hline & 5.5 & & & & 3 \\
\hline & $3 . E+$ & 7. E & 3. & $2 . E+04 / 3 . E$ & $2 . E+04 / 3 . E$ \\
\hline & & & & $4] 3 . E+04 / 2$ & +04 \\
\hline & $2 . E+17 / 1 . E+17$ & $3 . E+06 / 2 . E+$ & $E+03 / 5 . E+04$ & $42 . E+04 / 3 . E+04$ & $2 . E+04 / 4 . E+04$ \\
\hline & & & & & $8 . E+03 / 3 . E-$ \\
\hline
\end{tabular}

It can be seen that the best results as well as the lowest condition numbers correspond to the Chebyshev polynomials.

\section{Conclusions}

Our experiences can be summarized as follows.

- By the use of orthogonal polynomial bases the condition numbers of the basis matrices can be reduced dramatically. The second-kind Chebyhsev polynomials have performed best (see Example 1). 
- Sometimes the MDMP cannot be solved with monomial bases, however the orthogonal bases yield useful solutions. (see Example 3).

- The solution algorithm of Section 3 detects the infeasibilities even if they are under the numerical tolerances of the solver. The solver yields the result through the subscripts of the optimal basis, without numerical difficulties. Then the high precision arithmetic can check the solution. (see Example 5).

Until now, most results in connection with the MDMP have been about dual feasible basis structures. They have provided us with bounds on the objective function value. These bounds are very robust numerically, however they can be far from the optimum. On the other hand, the knowledge of dual feasible bases assumes some conditions on the function $f(\boldsymbol{z})$.

This paper has presented a different way of the numerical solution of the MDMP, without any assumption on the objective function. The computational experiments show that our method is substantially more stable numerically than the regular solution algorithms. Furthermore, it usually yields the optimum or a bound very close to the optimum value of the objective function.

Acknowledgements The European Union and the European Social Fund have provided financial support to the project under the grant agreement no. TÁMOP 4.2.1./B-09/1/KMR2010-0003.

\section{References}

1. Blyth, M. G., H. Luo and C. Pozrikidis. 2006. A comparison of interpolation grids over the triangle or the tetrahedron. J Eng Math 56 263-272.

2. Farouki, R. T. 2000. Legendre-Bernstein basis transformations. Journal of Computational and Applied Mathematics 119 145-160.

3. Gautschi, W. 1968. Construction of Gauss-Christoffel Quadrature Formulas. Mathematics of Computation 22(102) 251-270.

4. Gautschi, W. 1983. The Condition of Vandermonde-like Matrices Involving Orthogonal Polynomials. Linear Algebra and its Applications 52/53 293-300.

5. ILOG CPLEX 9. 2010. http://www-01.ibm.com/software/integration/optimization/cplex/

6. Li, R-C. 2006. Asymptotically Optimal Lower Bounds For the Condition Number of a Real Vandermonde Matrix. SIAM Journal on Matrix Analysis and Applications 28(3) 829-844.

7. Lyche, T. and J. M. Peña. 2004. Optimally stable multivariate bases. Advances in Computational Mathematics 20 149-159.

8. Lyche, T. and K. Scherer. 2000. On the p-norm condition number of the multivariate triangular Bernstein basis. Journal of Computational and Applied Mathematics 119 259273.

9. Lyche, T. and K. Scherer. 2002. On the $L_{1}$-Condition Number of the Univariate Bernstein Basis. Constr. Approx. 18 503-528.

10. Hou, X. and A. Prékopa. 2007. Monge Property and Bounding Multivariate Probability Distribution Functions with Given Marginals and Covariances. SIAM Journal on Optimization 18 138-155.

11. Mádi-Nagy, G. 2005. A method to find the best bounds in a multivariate discrete moment problem if the basis structure is given. Studia Scientiarum Mathematicarum Hungarica 42 (2) 207-226.

12. Mádi-Nagy, G. 2009. On Multivariate Discrete Moment Problems: Generalization of the Bivariate Min Algorithm for Higher Dimensions. SIAM Journal on Optimization 19(4) 1781-1806.

13. Mádi-Nagy, G. and A. Prékopa. 2004. On Multivariate Discrete Moment Problems and their Applications to Bounding Expectations and Probabilities. Mathematics of Operations Research 29 (2) 229-258. 
14. Mádi-Nagy, G. and A. Prékopa. 2007. Bounding Expectations of Functions of Random Vectors with Given Marginals and some Moments: Applications of the Multivariate Discrete Moment Problem. RUTCOR Research Report 11-2007.

15. Prékopa, A. 1988. Boole-Bonferroni Inequalities and Linear Programming. Operations Research 36 (1) 145-162.

16. Prékopa, A. 1990a. Sharp bounds on probabilities using linear programming. Operations Research 38 227-239.

17. Prékopa, A. 1990b. The discrete moment problem and Linear Programming. Discrete Applied Mathematics 27 235-254.

18. Prékopa, A. 1992. Inequalities on Expectations Based on the Knowledge of Multivariate Moments. M. Shaked and Y.L. Tong, eds., Stochastic Inequalities. Institute of Mathematical Statistics, Lecture Notes - Monograph Series, Vol 22, 309-331.

19. Prékopa, A. 1998. Bounds on Probabilities and Expectations Using Multivariate Moments of Discrete Distributions. Studia Scientiarum Mathematicarum Hungarica 34 349-378.

20. Prékopa, A. 2000. On Multivariate Discrete Higher Order Convex Functions and their Applications. RUTCOR Research Report 39-2000. Also in: Proceedings of the Sixth International Conference on Generalized Convexity and Monotonicity, Karlovasi, Samos, Greece, August 29 - September 2, to appear.

21. Prékopa, A. and G. Mádi-Nagy. 2008. A Class of Multiattribute Utility Functions. Economic Theory 34 (3) 591-602.

22. Samuels, S.M. and W.J. Studden. 1989. Bonferroni-Type Probability Bounds as an Application of the Theory of Tchebycheff System. Probability, Statistics and Mathematics, Papers in Honor of Samuel Karlin, Academic Press, 271-289.

23. Skeel, R. D. 1979. Scaling for Numerical Stability in Gaussian Elimination. Journal of the Association for Computing Machinery, 26(3) 494-526.

24. Wolfram's Mathematica 2010. http://www.wolfram.com/ 